\title{
Inferring surface heat flux distributions guided by a global seismic model: particular application to Antarctica
}

\author{
Nikolai M. Shapiro*, Michael H. Ritzwoller \\ Department of Physics, Center for Imaging the Earth's Interior, University of Colorado, Campus Box 390, Boulder, CO 80309, USA
}

Received 12 November 2003; received in revised form 1 April 2004; accepted 9 April 2004

\begin{abstract}
We present a method that uses a global seismic model of the crust and upper mantle to guide the extrapolation of existing heat-flow measurements to regions where such measurements are rare or absent. For any chosen spatial point on the globe, the procedure generates a histogram of heat-flow values determined from existing measurements obtained from regions that are structurally similar to the target point. The inferred histograms are based on a "structural similarity functional", which is introduced to quantify the structural analogy between different regions. We apply this procedure world-wide using the global heat-flow data base of Pollack et al. [Rev. Geophys. 31 (1993) 267] guided by an update of the 3-D shear velocity model of the crust and uppermost mantle of Shapiro and Ritzwoller [Geophys. J. Int. 51 (2002) 88]. The method results in an inferred probability distribution for the heat flux for each geographical region of interest. These distributions are strongly non-Gaussian, but are well approximated by the log-logistic distribution which is completely specified by two parameters. The inferred distributions agree well with observed distributions of heat flux taken in 300-km radius circles regionally in numerous locations. Particular attention is drawn to the inferred surface heat flux distributions across Antarctica, where direct measurements are rare but information about heat flow may be needed to help understand the dynamics of the Antarctic ice sheets and ice streams. Mean heat flow in West Antarctica is expected to be nearly three times higher than in East Antarctica and much more variable. This high heat flow may affect the dynamics of West Antarctic ice streams and the stability of the West Antarctic Ice Sheet. (C) 2004 Elsevier B.V. All rights reserved.
\end{abstract}

Keywords: heat flow; seismic tomographic model; structural similarity; ice sheets

\section{Introduction}

Heat flow measured at the Earth's surface is related to the temperatures of the Earth's crust and upper mantle and therefore provides valuable information about the structure and dynamics of the Earth's interior. Heat flow may also have an impor-

* Corresponding author. Tel.: +1-303-492-5243; fax: +1-303492-7935.

E-mail address: nshapiro@ciei.colorado.edu (N.M. Shapiro). tant effect in the dynamics of the ice sheets which are largely controlled by fast moving ice streams $[3,4]$ whose efficiency is affected by the lubricating effect of water in a weak basal layer. The melting/ freezing rate at the bottom of the ice depends on the sub-glacial geological conditions, including sub-glacial geothermal flux [5-7].

There are thousands of heat-flow measurements that have been obtained in a variety of tectonic regimes both within continents and oceans [1]. The distribution of these observations, however, is very 
inhomogeneous, having been obtained dominantly in the Northern hemisphere within North America and Europe (Fig. 1). In particular, heat-flow information across Antarctica is exceedingly rare because measurement of heat flow at the base of the Antarctic ice sheets is technically challenging.

Our purpose is to extrapolate existing heat-flow data accumulated over the entire globe and to infer heat-flow distributions for regions in which direct heat-flow measurements are rare or entirely absent. This will result in a smooth global heat-flow map that may prove useful in improving future seismic models $[8,9]$. Of particular interest is the map of geothermal flux across Antarctica.

There are several possible approaches to heat-flow extrapolation. Some methods use a tectonic regionalization to summarize known heat-flow information by tectonic type, and then apply the results to Antarctica. Rough regionalizations have been used to produce characteristic heat-flow values for continental tectonic regimes [1,10-12] and in oceans, average heat flow has been computed as a function of sea-floor age $[13,14]$. This approach is not ideal for Antarctica because Antarctic tectonics is not well understood. A second method that has some merit would be to predict heat flow directly from a 3-D seismic model. The merit derives from the fact that the vertical seismic velocity gradient in the mantle is related to the temperature gradient which controls the mantle component of heat flow [8,15-17]. Demerits include the necessity of introducing the crustal contribution to heat flow through the decay of radioactive elements in some way, the fact that heat-flow information from elsewhere in the world would not be explicitly utilized, and the confounding effects of composition, mantle volatiles, and anelasticity. Shapiro and Ritzwoller [8] argue that for seismic models to predict the mantle component of heat flow faithfully would require the imposition of physical constraints on the inversion which have not yet been applied systematically.

We aim to merge these two alternative methods in a way that will accentuate their strengths and minimize their weaknesses. Our approach is to use structural, as opposed to tectonic, analogies between parts of Antarctica and regions elsewhere in the world where heatflow measurements are available. The structural analogies are defined by a recent 3-D seismic model of the crust and uppermost mantle and are based on two implicit assumptions. First, we assume that seismic velocities are related to temperatures in the mantle and, therefore, to the mantle component of heat flow. Global seismic models have been clearly observed to be strongly correlated with mantle temperatures, on average. Continental tectonic regions and young oceanic regions, believed to be warm, are slow. Shields, continental platforms, and old oceanic lithosphere, believed to cold, are fast. In addition, seismic models are reconcilable quantitatively with surface heat-flow measurements over broad regions $[8,9]$. A caveat here is the possible effect of mantle composition (specifically, the volatile content) on seismic velocities in some regions. Second, we assume that crustal radioactive heat production is similar in regions with similar

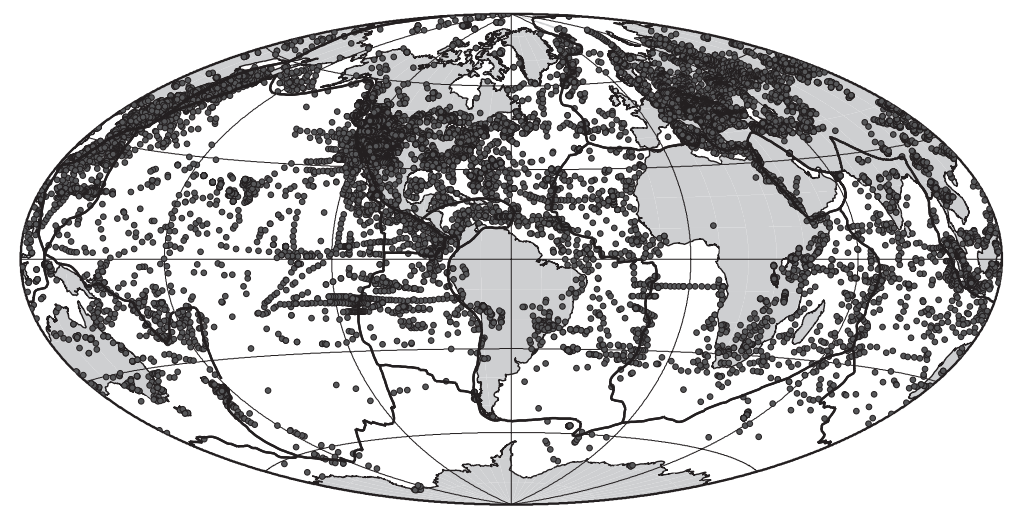

Fig. 1. Distribution of the heat-flow data from the data base of Pollack et al. [1]. Here and after, thick black lines show the plate boundaries. 
crustal structures. This is a more questionable assumption because the relation between seismic velocities and the concentration of radioactive elements remains poorly understood. It is, however, reasonable to expect that thick continental crust will produce more internal heat than thin oceanic crust, which implies that crustal composition and thickness are important variables. Sedimentary thickness is also important because of the concentration of radioactive elements in sediments. However, it is difficult to use this variable at global scales because of the non-uniform quality of the global sedimentary models. Overall, we believe that structural analogies have a more substantial physical basis than traditional analogies based on tectonic age observed at the surface. The structural analogy is specially advantageous for Antarctica where the surface geology is poorly known across large areas covered by the ice sheets. As described later in this paper, we formalize the structural analogy in terms of a "structural similarity functional" and develop a procedure that extrapolates existing heat-flow data to regions in which direct heat-flow measurements are rare or entirely absent.

Heat-flow observations vary considerably at length scales comparable to the lateral resolution of a global seismic model $[1,18]$. The method of extrapolating heat-flow measurements that we describe here accounts for this variability by producing inferred distributions of surface heat flux, rather than a single value at each nodal point on the globe. The procedure, therefore, does not provide a deterministic prediction, but rather at each geographical location presents a probability distribution for the likely heatflow values.

\section{The seismic model and structural similarity}

Seismic models based on surface waves are most suitable to establish structural similarities between different parts of the Earth because they provide relatively homogeneous coverage over the whole Earth and relatively good resolution in the crust and in the uppermost mantle which are the depths most directly related to surface heat flux. We use an update of the 3-D shear velocity model of Shapiro and Ritzwoller [2]. The data used to construct this model consist of a large set of dispersion curves for broad-band Rayleigh and Love wave group [19,20] and phase $[21,22]$ velocities. Period-specific dispersion maps were constructed with "diffraction tomography", which is based on a physical model of the surface-wave lateral sensitivity kernels [23]. These dispersion maps are subjected to a nonlinear MonteCarlo inversion that produces an ensemble of acceptable shear velocity models at all points on a $2 \times 2^{\circ}$ geographical grid world-wide [2].

Three features of this model make it particularly appropriate as a basis to infer structural similarity world-wide. First, the large number of short-period group-velocity measurements used in the construction of this model improves the vertical resolution of the model significantly compared to traditional surface-wave models based on phase-velocity measurements alone. Second, this model is based on a particularly large data set of surface-wave paths across Antarctica [20]. Third, because the model is based on a Monte-Carlo inversion, it has uncertainties, which we will need in the following.

We quantify the concept of structural similarity between two surface locations $x_{1}$ and $x_{2}$ by introducing the "structural similarity functional", $S\left(x_{1}, x_{2}\right)$. Although its definition is ad-hoc, the functional must satisfy certain conditions. For example, two points should be deemed perfectly similar when the shear velocity profiles directly beneath these points are identical and the points must be dissimilar when the corresponding profiles differ significantly. Here, we define the similarity functional as composed of two terms:

$$
\begin{aligned}
S\left(x_{1}, x_{2}\right)= & \left(1-w_{\text {Moho }}\right) S^{\beta}\left(x_{1}, x_{2}\right) \\
& +w_{\text {Moho }} S^{\text {moho }}\left(x_{1}, x_{2}\right) .
\end{aligned}
$$

The coefficient $w_{\text {Moho }}$ regulates the relative contribution between similarity in velocity structure and in crustal thickness. Similarity in shear velocity is quantified by $S^{\beta}\left(x_{1}, x_{2}\right)$, the weighted difference between the shear velocity profiles $\beta_{1}(z)$ and $\beta_{2}(z)$ at locations $x_{1}$ and $x_{2}$ :

$$
S^{\beta}\left(x_{1}, x_{2}\right)=\frac{\int_{z_{1}}^{z_{2}} w(z)\left(\frac{\left|\beta_{2}(z)-\beta_{1}(z)\right|}{u(z)}\right) \mathrm{d} z}{\int_{z_{1}}^{z_{2}} w(z) \mathrm{d} z},
$$


where $z$ is depth, the limits of the integral are 0 and 300 $\mathrm{km}, u(z)$ is the global average uncertainty of the seismic model estimated with the Monte-Carlo inversion, and $w(z)$ is a depth-dependent weight function. Fig. 2a shows the depth-dependent weight function and Fig. $2 \mathrm{~b}$ shows the estimated average uncertainty in the shear velocity model. As a result of these choices, the term $S^{\beta}\left(x_{1}, x_{2}\right)$ is dominated by the mantle part of the model. The second term $S^{\text {moho }}\left(x_{1}, x_{2}\right)$ is introduced to account for differences in crustal thickness between points $x_{1}$ and $x_{2}, Z_{1}^{\text {moho }}$ and $Z_{2}^{\text {moho }}$ :

$S^{\text {moho }}\left(x_{1}, x_{2}\right)=\frac{\left|Z_{1}^{\text {moho }}-Z_{2}^{\text {moho }}\right|}{\sigma_{\text {moho }}}$.

We use $w_{\text {Moho }}$ in the range between 0.25 and 0.5 and $\sigma_{\text {moho }}=10 \mathrm{~km}$, which effectively discriminates between oceanic and continental crust. Finally, note that the similarity functional depends strongly on crustal thickness, but upper-crustal velocities are significantly down-weighted (Fig. 2a). This weighting of crustal parameters follows from the vertical resolution of the global model. Crustal thickness is relatively well constrained, but model resolution deteriorates in the upper crust.

With similarity defined by Eq. (1), if $S\left(x_{1}, x_{2}\right)<1$, the depth-averaged difference between the two shear velocity profiles will be smaller than the average uncertainty in the model, so the points $x_{1}$ and $x_{2}$ will be considered to be similar. When $S\left(x_{1}, x_{2}\right)>1$, the difference between the profiles will be deemed significant and the two points will not be considered to be similar. Fig. $2 \mathrm{c}$ and d display maps of the similarity functional for two points in Antarctica. The one in East Antarctica (84S, 90E) is similar to old cratonic regions
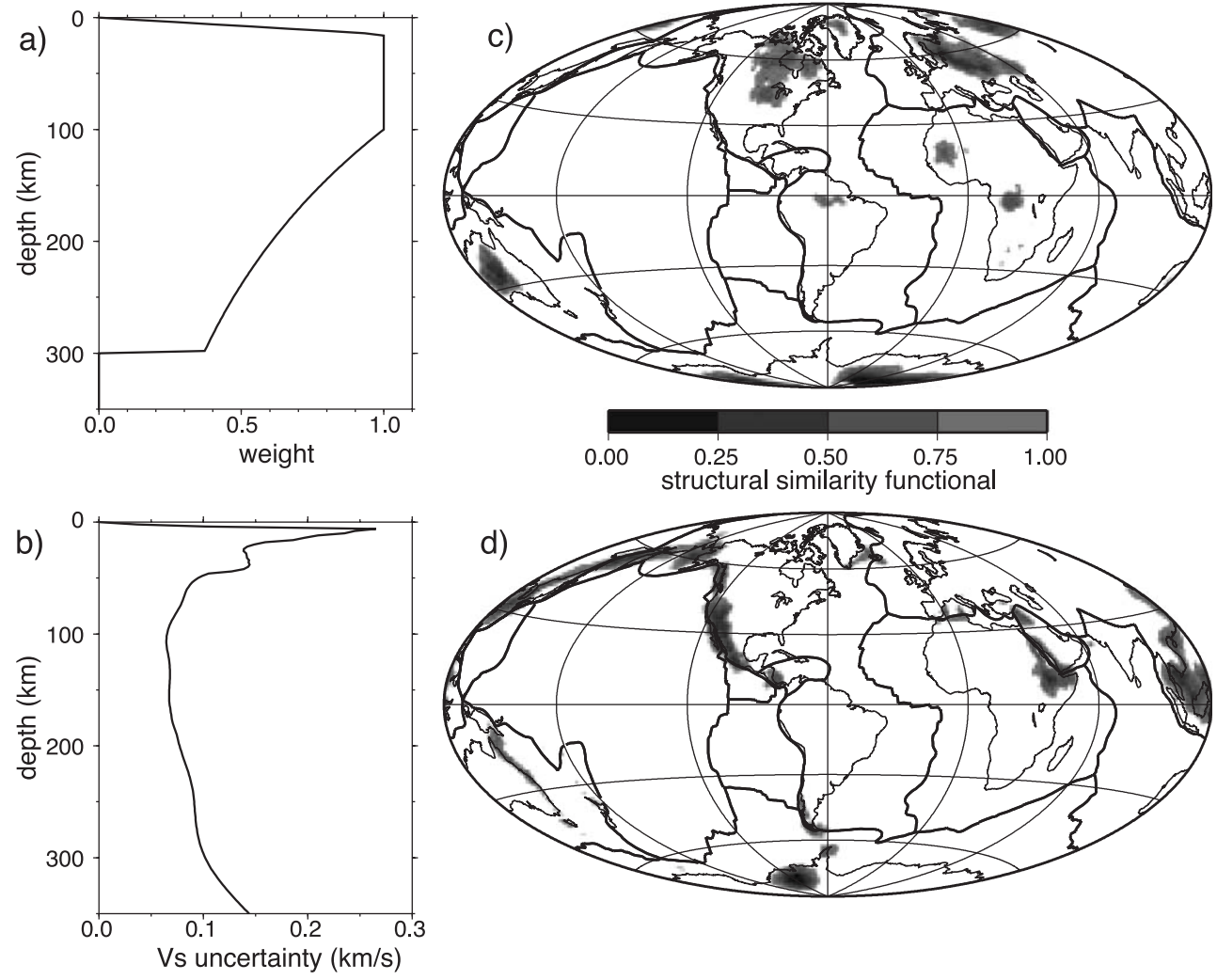

Fig. 2. (a) Weights $w(z)$ used in Eq. (2) to define the structural similarity functional, $S^{\beta}$. (b) Model uncertainty $u(z)$ used in Eq. (2). (c) The similarity functional for a location in East Antarctica (84S, 90E). Similarity is greatest for a value of 0; i.e., the darker regions, which are predominantly near shields and continental platforms. (d) The similarity functional for a location in West Antarctica (78S, 110W). West Antarctica is most similar to regions that have undergone recent tectonic deformation with relatively thin crust. 
(e.g., Canadian craton, East-European platform, Siberian craton, West-African craton) consistent with tectonic reconstructions showing East Antarctica to be a fragment of the old supercontinents Rodinia and Gondwana [24,25]. West Antarctic tectonics is dominated by the West Antarctic Rift System where active volcanism and seismicity have been discovered [5,2629]. Therefore, it is not surprising that the similarity functional identifies the point in West Antarctica (78S, $110 \mathrm{~W})$ to be similar to continental regions with relatively young extensional tectonics (e.g., Western North America, Iceland, Red Sea, Eastern Turkey, Western Mediterranean).

\section{Variability of heat-flow observations}

The lateral resolution of the global shear velocity model of Shapiro and Ritzwoller [2] across Antarctica is between 600 and $1000 \mathrm{~km}$. Therefore, extrapolation of heat-flow measurements based on this model will provide heat-flow estimates averaged across areas of this scale. The variability of heat-flow measurements over areas of this size is very large. For example, Fig. 3 shows histograms of heat-flow measurements in four different tectonic settings: an old continental platform, a young tectonic continent, an old ocean, and very young ocean near a mid-oceanic ridge. At each location, we selected all heat-flow measurements from the global data base [1] within a $300-\mathrm{km}$ radius circle and computed the histograms shown in Fig. 3. The resulting distributions are very broad, especially in regions with a high average heat flow. Similarly broad heat-flow distributions for different geological units have been found by Pollack et al. [1] with the variability increasing with the average heat flow (Fig. 4).

A variety of physical mechanisms contribute to the strong lateral variability of the geothermal flux; e.g.,
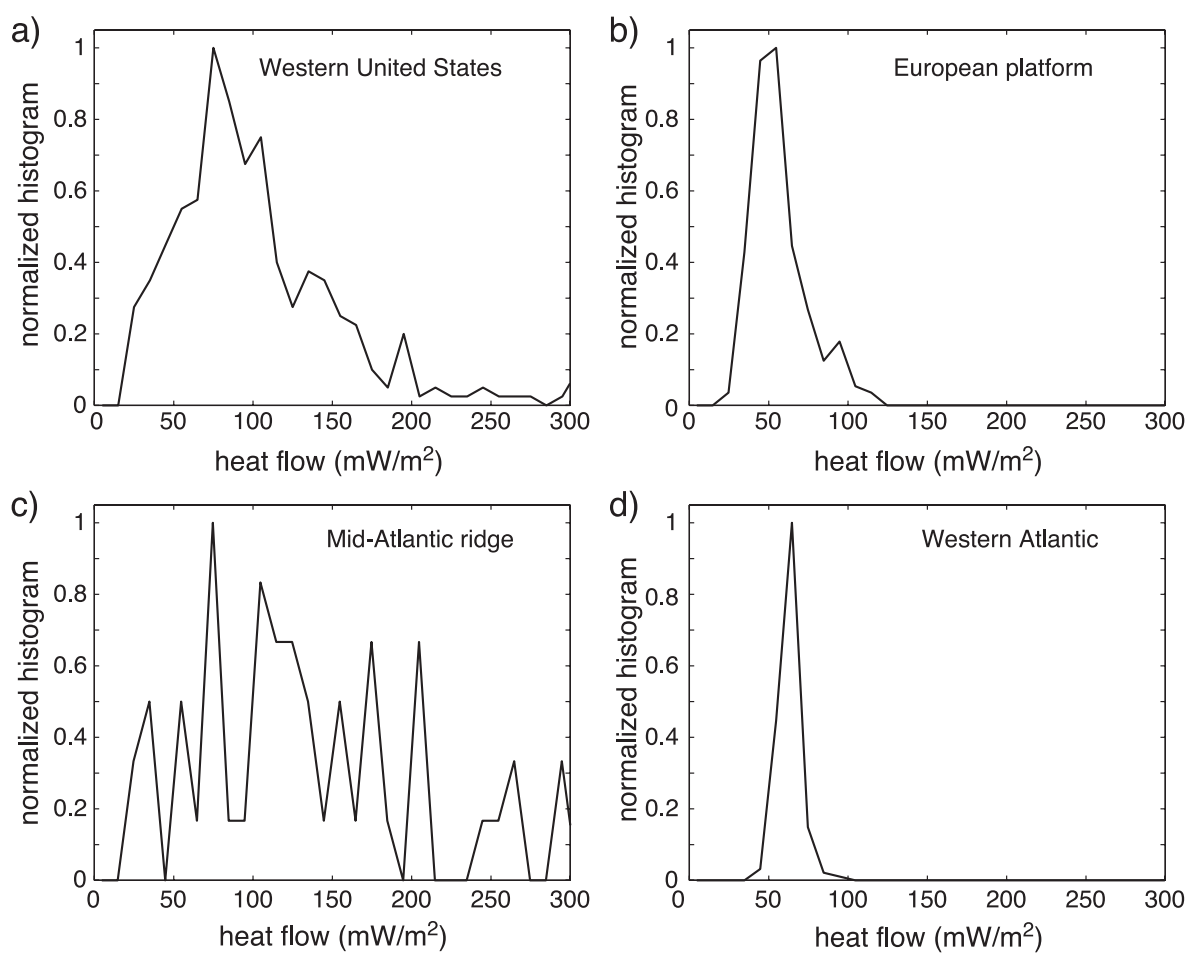

Fig. 3. Local histograms of observed surface heat flow taken from the data base of Pollack et al. [1] within $300 \mathrm{~km}$ of the specified location. (a) Western United States $(40 \mathrm{~N}, 120 \mathrm{~W})$. Total number of heat-flow measurements is $N=344$, mean heat flux is $\bar{Q}=107.7 \mathrm{~mW} / \mathrm{m}^{2}$, and standard deviation $\sigma_{Q}=85.9 \mathrm{~mW} / \mathrm{m}^{2}$. (b) European platform $(50 \mathrm{~N}, 30 \mathrm{E}) . N=198, \bar{Q}=45.9 \mathrm{~mW} / \mathrm{m}^{2}, \sigma_{Q}=17.3 \mathrm{~mW} / \mathrm{m}^{2}$. (c) Mid-Atlantic ridge near Island $(62 \mathrm{~N}, 24 \mathrm{~W}) . N=57, \bar{Q}=135.9 \mathrm{~mW} / \mathrm{m}^{2}, \sigma_{Q}=84.9 \mathrm{~mW} / \mathrm{m}^{2}$. (d) Western Atlantic $(32 \mathrm{~N}, 68 \mathrm{~W}) . N=156, \overline{\mathrm{Q}}=52.4 \mathrm{~mW} / \mathrm{m}^{2}$, $\sigma_{Q}=6.4 \mathrm{~mW} / \mathrm{m}^{2}$. 


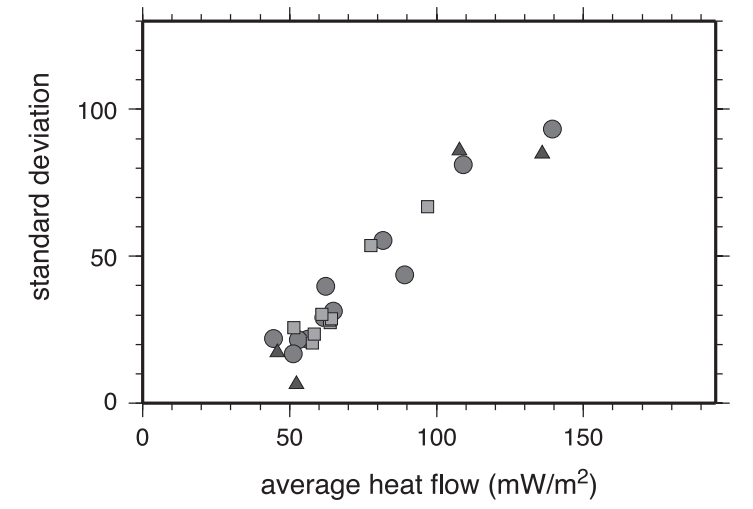

Fig. 4. Standard deviation of observed surface heat flux, $\sigma_{Q}$, plotted as function of the mean observed heat flux, $\bar{Q}$. Values from the four locations presented in Fig. 3 are shown with triangles. Circles and squares show the values estimated by Pollack et al. [1] for oceanic and continental tectonic units, respectively.

variations in the mantle geotherm, heterogeneous distribution of radioactive heat sources in the Earth's crust, hydrothermal circulation in the shallow crust. Hydrothermal circulation is expected to be more intense in tectonically active regions and may contribute to the increase in the standard deviation of heat-flow measurements with an increase in the average heat flow, as shown in Fig. 4. Although investigating the physical mechanisms that produce the large variability of the heat-flow observations is beyond the scope of this paper, it is important for the methods that extrapolate heat-flow information to account for this variability. The method, therefore, should not provide a deterministic estimate of surface heat flux, but rather should generate a probability distribution for the expected heat flow at each location averaged over a region of specified size.

\section{Inferred distribution of surface heat flux}

We use the global surface heat-flow compilation of Pollack et al. [1] as the primary heat-flow data set. Fig. 1 identifies the measurement locations and Figs. 3 and 4 demonstrate the variability of the measurements at several locations.

In the following, we will refer to measured heatflow values as $Q$, to be distinguished from inferred heat flow or heat flow as a functional variable for which we will use $q$.

The inference of the distribution of surface heat flux is an extrapolation procedure guided by the notion of two-point structural similarity. Structural similarity is quantified by the similarity functional in the following way. For each target location $x_{0}$ where we wish to infer a heat-flow distribution, the similarity functional $S\left(x_{0}, x\right)$ defines the regions with similar seismic structure (as shown in Fig. 2c and d). The inferred heat flux distribution is computed from the heat-flow measurements within those regions of similarity. This is formally performed by introducing a similarity-based weight function:

$W(S)=\exp \left(-\frac{S^{2}}{2 \sigma_{\mathrm{S}}^{2}}\right)$

where $\sigma_{\mathrm{S}}$ is a free parameter defining the degree of smoothing. The weight $W\left[S\left(x_{0}, x_{n}\right)\right]$, defined by Eq.
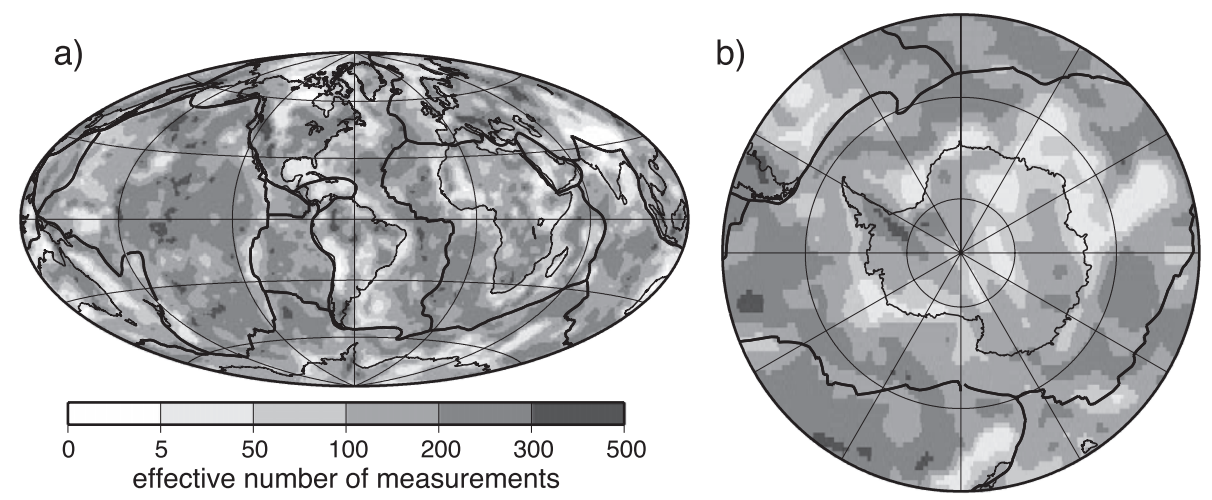

Fig. 5. Effective number of heat-flow measurements, $N_{\mathrm{e}}$ (Eq. (5)), to be applied at each spatial point. 
(4), is the effective number of heat-flow measurements deriving from locations $x_{n}$ that are deemed applicable to location $x_{0}$. The total effective number of measurements to be applied at location $x_{0}$ is:

$N_{\mathrm{e}}\left(x_{0}\right)=\sum_{n} W\left[S\left(x_{0}, x_{n}\right)\right]$,

where $n$ is an index over all heat-flow measurements $Q_{n}$. As shown in Fig. 5, there are a few areas where the effective number of measurements is small because of unusual crustal or mantle structure; e.g., the Bolivian Altiplano and Tibet because of anomalously thick crust.

Using the measurements from regions that are structurally similar to location $x_{0}$, the following equation estimates the heat-flow histogram that we assign to $x_{0}$ :

$\mathcal{H}\left(x_{0}, q \pm \mathrm{d} q\right)=\sum_{n} W\left[S\left(x_{0}, x_{n}\right)\right] B\left[Q_{n}, q \pm \mathrm{d} q\right]$,

where $Q_{n}$ is the measured heat flow at location $x_{n}$, the function $B\left[Q_{n}, q \pm \mathrm{d} q\right]=1$ if $Q_{n}$ lies between $q-\mathrm{d} q$
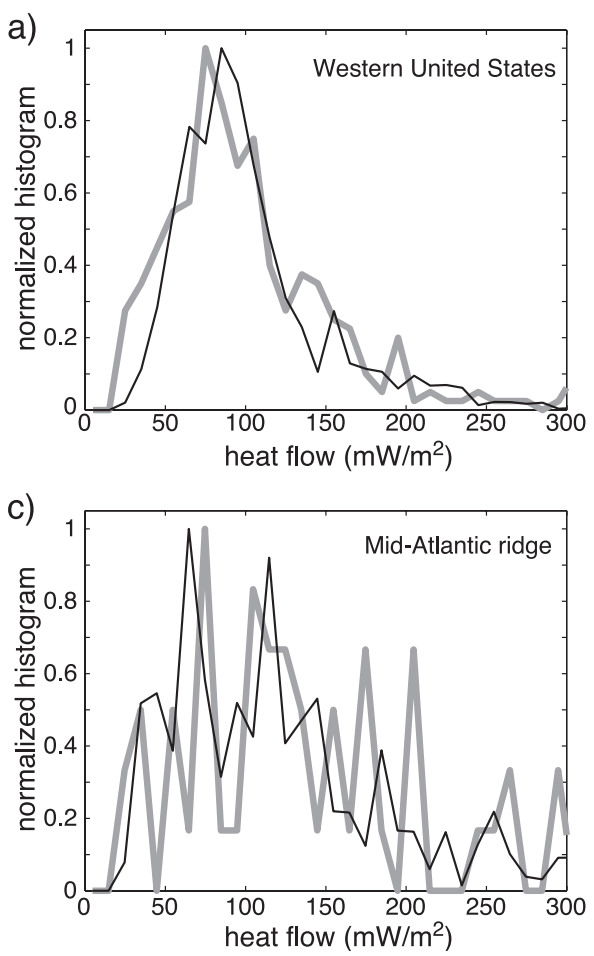

and $q+\mathrm{d} q$ and is 0 otherwise, and we set $\mathrm{d} q=2.5 \mathrm{~mW} /$ $\mathrm{m}^{2}$. The value of the histogram in the interval $q \pm \mathrm{d} q$ at location $x_{0}$, therefore, is a weighted sum of all measurements in structurally similar regions, where the weights are given by Eq. (4).

We interpret this histogram as a probability distribution or probability density function. Examples of inferred distributions at four locations, compared with the local heat-flow data, are shown in Fig. 6. In general, we see considerable agreement between the inferred (extrapolated) distributions and the local data, particularly at continental locations.

The inferred heat-flow distributions are also found to be fairly robust with respect to the choice of parameters defining the similarity functional. For example, varying the coefficients $\sigma_{\mathrm{S}}$ and $w_{\text {Moho }}$ by a factor of two affects the inferred distribution in continental areas very little, as Fig. 7a demonstrates. The affect is somewhat larger in old oceanic regions and is most significant near mid-oceanic ridges (Fig. 6c)
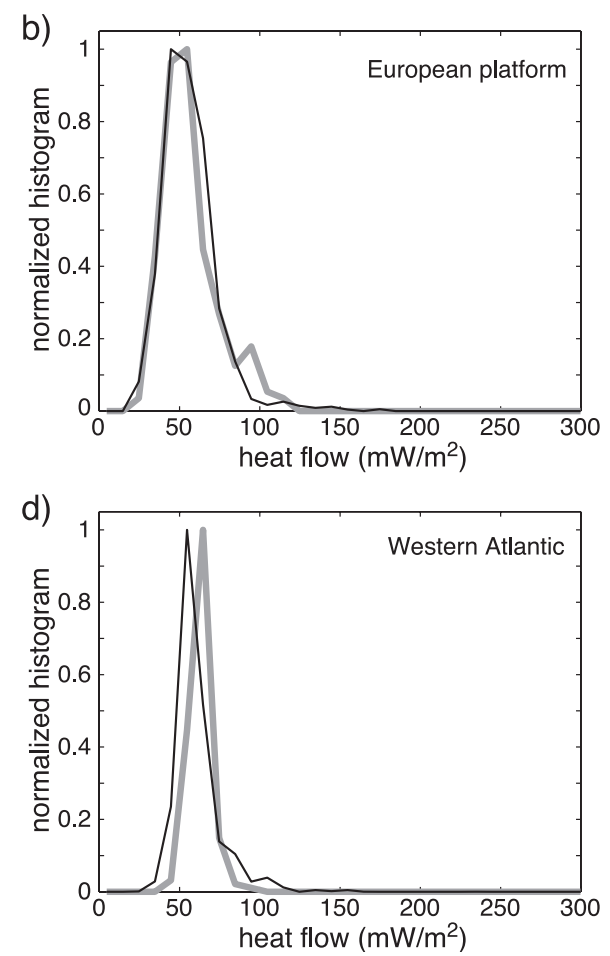

Fig. 6. Comparison of heat-flow histograms computed using local observations $\left(Q_{n}\right)$ obtained within $300 \mathrm{~km}$ of the specified locations, with the histograms $\mathcal{H}(q)$ inferred using Eq. (6). The four locations are specified in Fig. 3. Solid black lines are the inferred histograms and thick grey lines are the histograms constructed from local observations. The local data have been segregated and not included in computing the inferred histograms in each case. 

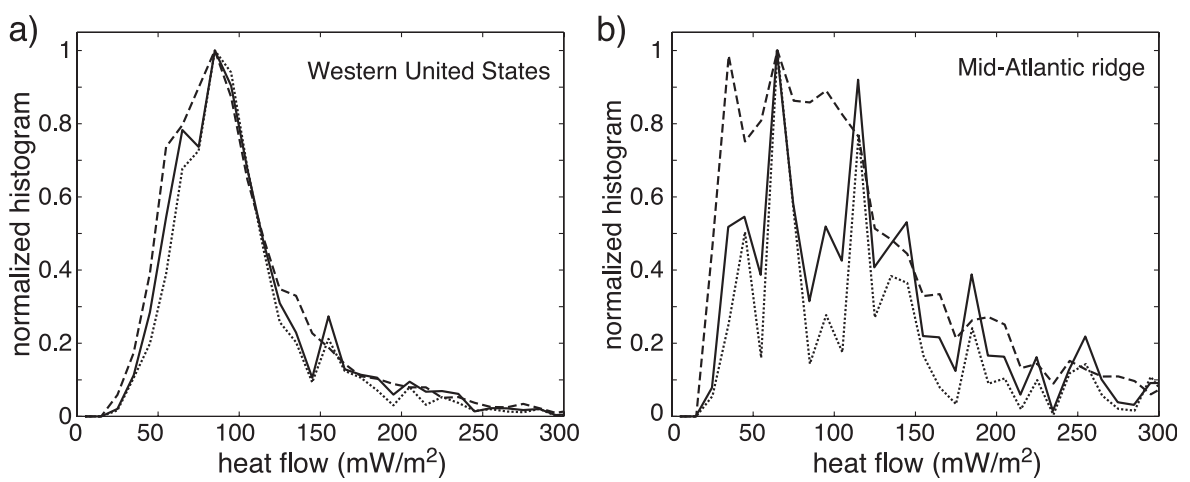

Fig. 7. Assessment of the effect of varying parameters in the similarity functional on the inferred histogram of surface heat flux, $\mathcal{H}(q)$, for two of the locations shown in Fig. 3. Solid black lines are the inferred histograms obtained with $w_{\text {Moho }}=0.5$ and $\sigma_{\mathrm{S}}=0.2$, dashed lines are the histograms obtained with $w_{\mathrm{Moho}}=0.5$ and $\sigma_{\mathrm{S}}=0.4$, and dotted lines are the histograms obtained with $w_{\mathrm{Moho}}=0.25$ and $\sigma_{\mathrm{S}}=0.2$. Histograms inferred within continents are robust relative to these choices, but histograms for young oceanic regions are more variable due to the higher variation of heat flux measurements near mid-ocean ridges.

where the variance of the heat-flow measurements is especially high presumably because of the considerable spatial variability of hydrothermal circulation $[1,18]$. Despite this variability, the inferred heat-flow distributions capture the principal properties of the expected distribution of local heat flux even in oceanic regions; e.g, the width of the distribution and heavy tail toward high values near oceanic ridges. Overall, the proposed method provides reasonable inferences of heat-flow histograms for a wide variety of tectonic settings.

Another test of the method is the ability to reproduce the nearly linear functional relationship between the local mean of heat flux measurements $\bar{Q}\left(x_{0}\right)$ and their standard deviation $\sigma_{Q}\left(x_{0}\right)$, seen, for example, in Fig. 4. The mean and standard deviation can be computed from the inferred distributions, or equivalently from the weighed sum over all heat-flow measurements world-wide obtained at locations $x_{n}$, where the weights derive from Eq. (4):

$\begin{aligned} \bar{q}\left(x_{0}\right) & =\frac{\sum_{n} W\left[S\left(x_{0}, x_{n}\right)\right] Q\left(x_{n}\right)}{\sum_{n} W\left[S\left(x_{0}, x_{n}\right)\right]}, \\ \sigma_{q}\left(x_{0}\right) & =\left\{\frac{\sum_{n} W\left[S\left(x_{0}, x_{n}\right)\right]\left[Q\left(x_{n}\right)-Q\left(x_{0}\right)\right]^{2}}{\sum_{n} W\left[S\left(x_{0}, x_{n}\right)\right]}\right\}^{1 / 2},\end{aligned}$

where $Q\left(x_{n}\right)$ are the observed heat-flow values.
Global maps of the mean and standard deviation of inferred surface heat flux estimated using Eqs. (7) and (8) are shown in Fig. 8. Here and later, we set $w_{\text {Moho }}=0.5$ and $\sigma_{\mathrm{S}}=0.2$. This choice of parameters is ad-hoc, but has been selected to discriminate between oceanic and continental regions while providing reasonable lateral resolution and keeping the effective number of measurements, $N_{\mathrm{e}}$, relatively high at most locations.

Heat flux is inferred to be low for old continental regions and maximizes on the mid-oceanic ridges and in regions that have undergone recent tectonic deformation. The inferred standard deviations follow the same pattern as the mean heat flow; i.e., they minimize in old continental regions and maximize along mid-oceanic ridges and in tectonic continental areas, such as West Antarctica. Fig. 9 shows that the inferred standard deviation is nearly a linear function of the inferred mean heat flux, similar to the relation from Pollack et al. [1].

\section{Parameterizing the distribution of heat flux}

Over most of the Earth, the inferred heat flux distribution can be well approximated with a simple parameterized curve. Fig. 10 shows an example where the inferred distribution of heat flow, $\mathcal{H}(q)$, is well represented with the loglogistic distribution whose probability density 

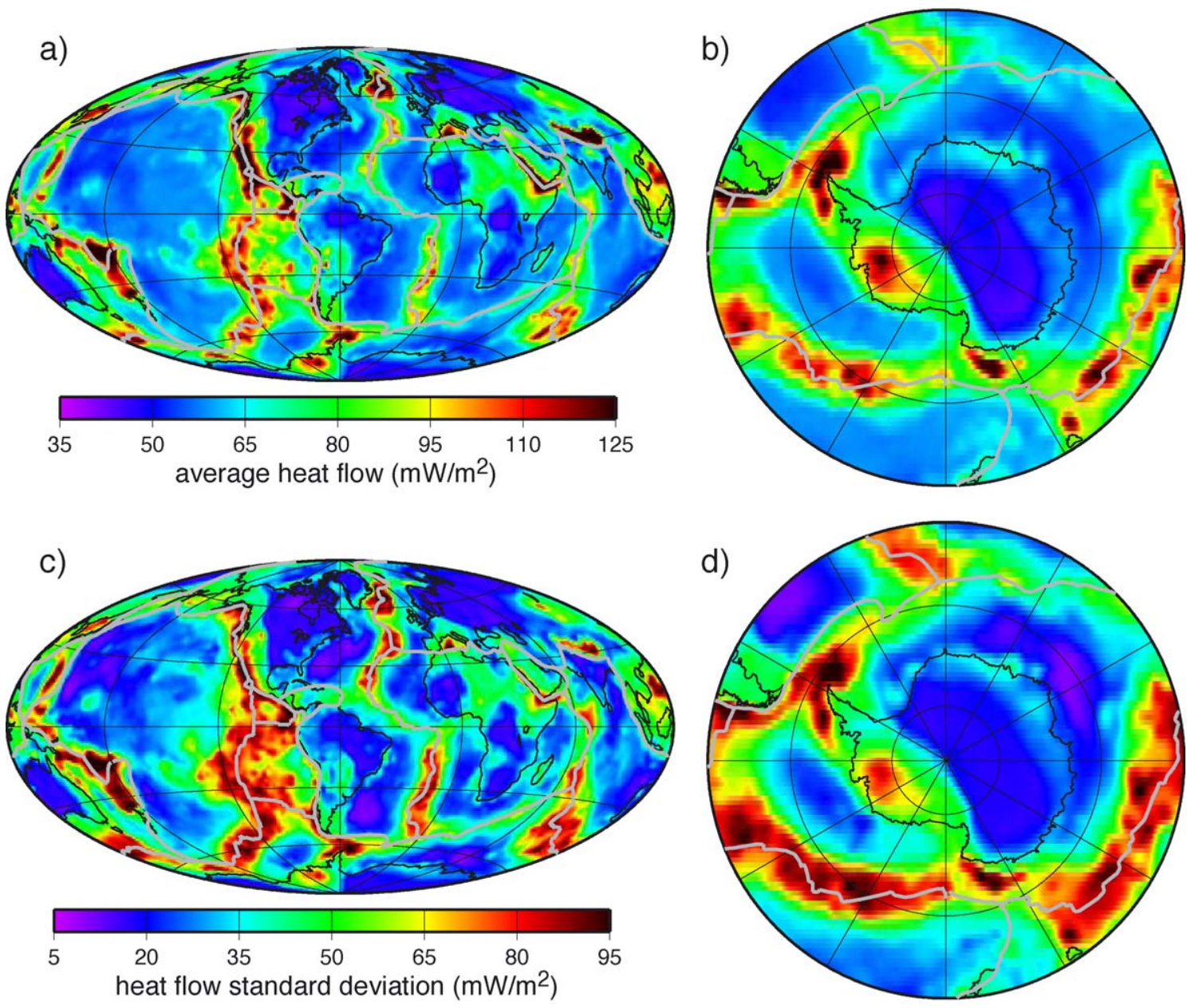

Fig. 8. (a and b) Mean of the inferred heat flux distribution, $\bar{q}$, estimated by Eq. (7). (c and d) Standard deviation of the inferred heat flux distribution, $\sigma_{q}$, estimated by Eq. (8).

function (PDF) is completely specified in terms of the local mean $(\bar{q})$ and an exponent $C$, as follows:

$\operatorname{PDF}(q)=N \frac{(\alpha q / \bar{q})^{C-1}}{\left[1+(\alpha q / \bar{q})^{C}\right]^{2}}$

where $N$ is a normalizing coefficient $N=\alpha C / \bar{q}, \alpha=(\pi / C)$ $\csc (\pi / C), \beta=(2 \pi / C) \csc (2 \pi / C)$, and the relationship between the mean and standard deviation is $\sigma_{q}=\bar{q}\left(\left(\beta-\alpha^{2}\right) / \alpha^{2}\right)^{1 / 2}$. As $C$ decreases, the distribution becomes increasingly heavy-tailed, and as $C$ increases, the distribution approaches a Gaussian. We are unclear why the log-logistic distribution approximates observed and inferred heat-flow histograms so well, whether it says something about the physics of heat flow or is simply related to the flexibility of the distribution.

East Antarctica is characterized by a heat flux distribution typical for stable continents, with a low average heat flow, whereas in West Antarctica the distribution is much broader with a significantly larger mean. The predicted heat-flow distributions are non-Gaussian, similar to distributions computed 


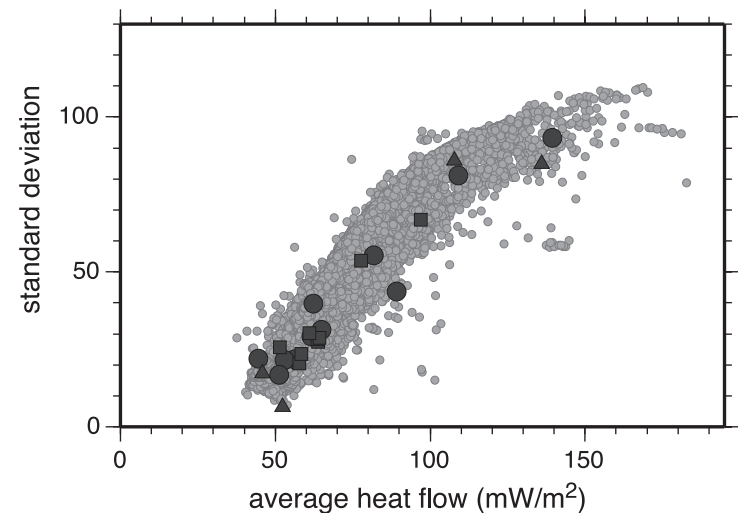

Fig. 9. Standard deviation of the inferred distribution of surface heat flux $\left(\sigma_{q}\right.$, Eq. (8)) plotted as function of the inferred mean of the distribution, $\bar{q}$, taken from Eq. (7), for all points on a $2^{\circ} \times 2^{\circ}$ global grid (small light circles). Values from the four locations shown in Fig. 3 indicated shown with dark triangles. Dark circles and squares show the values presented by Pollack et al. [1] for oceanic and continental tectonic units, respectively.

from the local data (Fig. 3) and to the global heatflow histograms of Pollack et al. [1].

\section{Discussion}

We have presented a method that uses a global seismic model of the crust and upper mantle to extrapolate existing heat-flow measurements to regions where such measurements are rare or absent. This method is based on the idea that regions that are structurally similar are expected to have similar levels of heat flow. The structural similarity is quantified by introducing a structural similarity functional. The method allows us to infer a probability distribution for the heat flux at each geographical location. These distributions are strongly non-Gaussian, but are well approximated by a simple parameterization, the loglogistic distribution which is completely specified with two parameters (Eq. (9)). They agree well with observed distributions of heat flux over much of the Earth.

The implementation of the proposed method is nonunique and depends on a number of ad-hoc choices such as the heat-flow data set, a particular global 3-D seismic model, the specific form of the model-based similarity functional, and the form of the corresponding similarity weight function. Those choices, however, are not completely arbitrary. The parameters that compose the similarity functional (in particular, relative contributions from the crust and from the mantle) were selected based on the resolution of the seismic model [2]. In addition, the inferred distributions of heat flux are relatively robust to variations in the choices of parameters that define the extrapolation method, particularly in continental areas.

An important application of the method presented here is to estimate the expected heat flux for Antarctica, which is needed as input into models of the dynamic evolution of the Antarctic Ice Sheet. A map of inferred mean heat-flow (Fig. 8) demonstrates substantial difference between East and West Antarctica, being almost three times higher in West Antarctica. This elevated heat flux may increase the rate of melting at the base of the ice and provide more efficient lubrication for fast moving ice streams. This mechanism may contribute to the instability of the West Antarctic Ice Sheet [30-32].

Mean heat flux shown in Fig. 8, however, does not reflect small-scale patterns caused by variations in hydrothermal circulation, volcanic activity, crustal radioactive heat production, etc. that may prove to be very important in modeling the behavior of Antarctic ice streams. The map of mean values also does

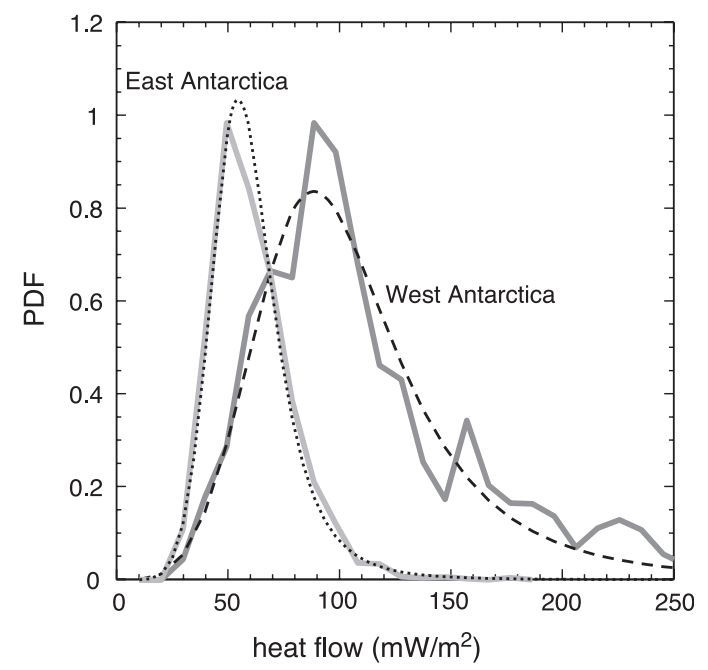

Fig. 10. Inferred histograms of surface heat flux estimated using Eq. (6) for a point in East Antarctica $(84 \mathrm{~S}, 90 \mathrm{E})$ and a point in West Antarctica $(78 \mathrm{~S}, 110 \mathrm{~W})$. The best-fitting log-logistic probability density functions are shown with dashed lines. For the West Antarctic location $C=4.0$ and $\bar{q}=107$, and for the East Antarctic location $C=5.5$ and $\bar{q}=57$. 
not provide a statistically meaningful realization of the heat-flow across Antarctica. A longer term challenge, therefore, will be to find ways to utilize the full inferred distribution rather than simply the mean of the distribution. Realistic heat-flow realizations may be obtained by generating at each geographical location random values that satisfy the particular loglogistic distribution at that location. Such random realizations must also simulate realistic spatial correlation of the heat flux. Developing the methods to generate such stochastic heat-flow realizations is, however, beyond the scope of the present paper.

Although concentration here has been placed on extrapolating heat-flow measurements to regions devoid of such information, the method we describe may also be useful for interpolation and to produce a smooth global map. Global heat-flow maps, such as the mean of the inferred heat flux distribution shown in Fig. 8, may also prove useful as constraints to improve future seismic models. Shapiro and Ritzwoller [8] discuss how the assimilation of heat-flow data into seismic inversions can improve the seismic model. A major problem is the uncertainty in the quality of a global model of heat flow, which the methods discussed in this paper may help to address. The construction of the inferred mean heat flux map presented here, therefore, may be seen as part of an iterative process in which the seismic model guides the inference of global heat flux which may in turn be fed-back to help refine the seismic model.

Continued improvements in seismic models and further refinements in the heat-flow data base will yield better inferences of surface heat flux in the future. Discussions are underway toward developing much improved seismic infrastructure on Antarctica, which promises great improvements in the resolution of the seismic model across the continent. Application of the estimated heat flux as a basal boundary condition in models of the dynamics of the Antarctic ice sheets will be particularly aided by improvements in the resolution of seismic models across Antarctica.

\section{Acknowledgements}

This work was inspired by conversations with Donald Blankenship and Slawek Tulaczyk at the Structure and Evolution of the Antarctic Plate 2003 community workshop in Boulder, CO in early March, 2003. We thank Scott King, Irina Artemieva, JeanClaude Mareschal, Saskia Goes, and two anonymous reviewers for criticism that helped to clarify this paper. The work was supported by a grant from the US National Science Foundation, NSF-OPP-0125848. [SK]

\section{References}

[1] H.N. Pollack, S.J. Hurter, J.R. Johnson, Heat flow from the Earth's interior: analysis of the global data set, Rev. Geophys. 31 (1993) 267-280.

[2] N.M. Shapiro, M.H. Ritzwoller, Monte-Carlo inversion for a global shear velocity model of the crust and upper mantle, Geophys. J. Int. 51 (2002) 88-105.

[3] J.L. Bamber, D.V. Vaughan, I. Joughin, Widespread complex flow in the interior of the Antarctic Ice Sheet, Science 287 (2000) $1248-1250$.

[4] E. Rignot, R.H. Thomas, Mass balance of polar ice sheets, Science 297 (2002) 1502-1506.

[5] D.D. Blankenship, R.E. Bell, S.M. Hodge, J.M. Brozena, J.C. Behrendt, C.A. Finn, Active volcanism beneath the West Antarctic Ice Sheet and implications for ice-sheet stability, Nature 361 (1993) 526-529.

[6] S. Anandakrishnan, D.D. Blankenship, R.B. Alley, P.L. Stoffa, Influence of sub-glacial geology on the position of a West Antarctic ice stream from seismic observations, Nature 394 (1998) 62-65.

[7] S. Tulaczyk, W.B. Kamb, H.F. Engelhardt, Basal mechanics of Ice Stream B, West Antarctica: 2. Undrained plastic bed model, J. Geophys. Res. 105 (2000) 483-494.

[8] N.M. Shapiro, M.H. Ritzwoller, Thermodynamics constraints on seismic inversions, Geophys. J. Int., in press.

[9] N.M. Shapiro, M.H. Ritzwoller, J.C. Mareschal, C. Jaupart, Lithospheric structure of the Canadian Shield inferred from inversion of surface-wave dispersion with thermodynamic a priori constraints, In: R. Wood, A. Curtis (Eds.), Geological Prior Information, Geol. Soc. London Spec. Publ., in press.

[10] A.A. Nyblade, H.N. Pollack, A global analysis of heat flow from Precambrian terrains: implications for the thermal structure of Archean and Proterozoic lithosphere, J. Geophys. Res. 98 (1993) $12207-12218$.

[11] R.L. Rudnick, W.F. McDonough, R.J. O'Connell, Thermal structure, thickness and composition of continental lithosphere, Chem. Geol. 145 (1998) 395-411.

[12] C. Jaupart, J.-C. Mareschal, The thermal structure and thickness of continental roots, Lithos 48 (1999) 93-114.

[13] B. Parsons, J.G. Sclater, An analysis of the variation of ocean floor bathymetry and heat flow with age, J. Geophys. Res. 82 (1997) 803-827.

[14] C.A. Stein, S. Stein, A model for the global variation in oceanic depth and heat flow with lithospheric age, Nature 359 (1992) $123-129$. 
[15] S.V. Sobolev, H. Zeyen, G. Stoll, F. Werling, R. Altherr, K. Fuchs, Upper mantle temperatures from teleseismic tomography of French Massif Central including effects of composition, mineral reactions, anharmonicity, anelasticity and partial melt, Earth Planet. Sci. Lett. 157 (1996) 193-207.

[16] S. Goes, R. Govers, R. Vacher, Shallow mantle temperatures under Europe from $\mathrm{P}$ and $\mathrm{S}$ wave tomography, J. Geophys. Res. 105 (2000) 11153-11169.

[17] A.H.E. Röhm, R. Snieder, S. Goes, J. Trampert, Thermal structure of continental upper mantle inferred from S-wave velocity and surface heat flow, Earth Planet. Sci. Lett. 181 (2000) 395-407.

[18] J.G. Sclater, C. Jaupart, D. Galson, The heat flow through oceanic and continental crust and the heat loss of the Earth, Rev. Geophys. 18 (1980) 269-311.

[19] M.H. Ritzwoller, A.L. Levshin, Eurasian surface wave tomography: group velocities, J. Geophys. Res. 103 (1998) 4839-4878.

[20] M.H. Ritzwoller, N.M. Shapiro, A.L. Levshin, G.M. Leahy, The structure of the crust and upper mantle beneath Antarctica and the surrounding oceans, J. Geophys. Res. 106 (2001) $30645-30670$.

[21] J. Trampert, J.H. Woodhouse, Global phase velocity maps of Love and Rayleigh waves between 40 and 150 s period, Geophys. J. Int. 122 (1995) 675-690.

[22] G. Ekström, J. Tromp, E.W.F. Larson, Measurements and global models of surface waves propagation, J. Geophys. Res. 102 (1997) 8137-8157.

[23] M.H. Ritzwoller, N.M. Shapiro, M.P. Barmin, A.L. Levshin, Global surface wave diffraction tomography, J. Geophys. Res. 107 (2002) 2335 (DOI: 10.1029/2002JB001777).
[24] I.W.D. Dalziel, Antarctica; a tale of two supercontinents? Annu. Rev. Earth Planet. Sci. 20 (1992) 501-526.

[25] W.A. Gose, M.A. Helper, J.N. Connelly, F.E. Hutson, I.W.D. Dalziel, Paleomagnetic data and $\mathrm{U}-\mathrm{Pb}$ isotopic age determinations from Coats Land, Antarctica: implications for late Proterozoic plate reconstructions, J. Geophys. Res. 102 (1997) 7887-7902.

[26] W.E. LeMasurier, Late Cenozoic volcanism on the Antarctic Plate-an overview, in: W.E. LeMasurier, J.W. Thomson (Eds.), Volcanoes of the Antarctic Plate and Southern Oceans, AGU Antarctic Research Series, vol. 48, 1990, pp. 1-17.

[27] J.C. Behrendt, C.A. Finn, D.D. Blankenship, R.E. Bell, Aeromagnetic evidence for a volcanic caldera(?) complex beneath the divide of the West Antarctic Ice Sheet, Geophys. Res. Lett. 25 (1998) 4385-4388

[28] J.C. Behrendt, Crustal and lithospheric structure of the West Antarctic Rift System from geophysical investigations - a review, Glob. Planet. Change 23 (1999) 25-44.

[29] J.P. Winberry, S. Anandakrishnan, Seismicity and neotectonics of West Antarctica, Geophys. Res. Lett. 30 (2003) DOI: 10.1029/2003GL018001.

[30] R. Bindschadler, Future of the West Antarctic Ice Sheet, Science 282 (1998) 428-429.

[31] M. Oppenheimer, Global warming and the stability of the West Antarctic Ice Sheet, Nature 393 (1998) 325-331.

[32] R.P. Sherer, A. Aldahan, S. Tulaczyk, G. Possnert, H. Engelhardt, B. Kamb, Pleistocene collapse of the West Antarctic Ice Sheet, Science 281 (1998) 82-85. 